\title{
La migración venezolana en el discurso periodístico
}

\section{Venezuelan Migration in Journalistic Discourse}

Recepción: 29/11/2020, revisión: 04/03/2021, aceptación: 11/03/2021, publicación: septiembre de 2021

(D) Ligia Adriana López Montenegro

https://revistas.uasb.edu.ec/index.php/uru

adrylopm@hotmail.com

Universidad Andina Simón Bolívar (Quito, Ecuador)

https://orcid.org/0000-0002-2999-0322

DOI: https://doi.org/10.32719/26312514.2021.4.10

\section{Resumen}

Las condiciones sociales, políticas y económicas de Venezuela han ocasionado que su población haya tomado la decisión de emigrar hacia otras naciones, entre ellas Ecuador. Al ser un tema que incide en los diferentes ámbitos sociales, ha sido abordado por diferentes medios de comunicación. Dentro del presente estudio se analizó cómo se construye el discurso periodístico del diario La Nación de la ciudad de Tulcán, en relación al proceso migratorio de la población venezolana hacia Ecuador, y de qué manera esto influye en la creación de representaciones sociales. La metodología del estudio se centró en la aplicación de la versión técnica comunicacional, a fin de profundizar en la problemática, el contexto y los actores alrededor del medio de comunicación. Además, se tomaron en cuenta los principios del análisis crítico del discurso periodístico para identificar las representaciones sociales presentes en lo relacionado con el tema migratorio; en total se analizaron cuatro notas informativas. Los resultados mostraron que el enfoque, el lenguaje, la intencionalidad y la producción discursiva del tema migratorio generan en la audiencia la creación de representaciones sociales parcializadas sobre la realidad de los migrantes, invisibilizando y desconociendo el contexto de su situación. Por esta razón es importante que los medios de comunicación cuenten con instrumentos que los ayuden a producir información que permita a la audiencia entender la migración desde sus diferentes dimensiones.

\section{Abstract}

The social, political and economic conditions of Venezuela have caused its population to have made the decision to emigrate to other nations, including Ecuador. As it is an issue that affects different social spheres, it has been addressed by different media. Within the present study, it was analyzed how the journalistic discourse of the newspaper La Nacion is constructed, in relation to the migratory process of the Venezuelan population to Ecuador and how this influences the creation of social representations? The study methodology focused on the application of the Technical Communicational Version, in order to delve into the problem, the context and the actors around the communication medium, in addition, the principles of critical discourse analysis were taken into account to identify the representations present in the journalistic discourse related to the immigration issue, a total of 4 informative notes were analyzed. The results showed that the 
approach, language, intentionality and discursive production of the migration issue will generate in the audience the creation of biased social representations about the reality of migrants, making them invisible and ignoring the context of their situation. For this reason, it is important that the media have instruments that help them produce an informative production that allows the audience to understand migration from its different dimensions.

\section{Palabras clave $\cdot$ Keywords}

Movilidad humana, representaciones sociales, análisis del discurso, medios de comunicación, noticia, versión técnica comunicacional.

Human mobility, social representations, discourse analysis, media, news, technical communication version.

\section{Introducción}

La migración es una actividad propia de los seres humanos que ha formado parte de sus formas de vida a lo largo de la historia. Se trata de una medida que tiene como finalidad enfrentar distintas circunstancias que se presentan dentro de la estructura social. En el caso de Venezuela, se ha convertido en opción para una población inmersa en problemáticas de carácter político, social y económico que atentan contra su bienestar, seguridad y calidad de vida. En este contexto, Ecuador se ha convertido en uno de los principales destinos en los cuales los migrantes se establecen con el afán de encontrar y construir nuevas oportunidades.

Debido a que se trata de una problemática que ha tenido incidencia a escala global, la han abordado los medios de comunicación alrededor del mundo, relatando a través de televisión, radio y prensa historias que intentar ser un reflejo de la realidad que viven los ciudadanos venezolanos. En este proceso de creación y difusión de información, se observa la manera en que el discurso periodístico ha contribuido a la implantación de imaginarios y representaciones sociales que asocian a los migrantes venezolanos con hechos violentos, delictivos y de inseguridad, los cuales no son necesariamente un reflejo veraz de la realidad.

Por consiguiente, el tema que interesa abordar es la construcción de representaciones sociales en el discurso mediático sobre el proceso migratorio de la población venezolana hacia Ecuador. Se considerará la perspectiva del periódico La Nación de la ciudad de Tulcán, a fin de determinar su línea editorial y cómo su discurso da paso a la conformación de imaginarios en torno al tema de la movilidad. La temporalidad definida corresponde a los meses de enero a marzo del año 2019; cabe tener en cuenta que su edición impresa circula solo los días domingos.

El análisis, desde una perspectiva comunicacional estratégica, permite indagar sobre el papel que juegan los mass media en la construcción de representaciones sociales que se instalan el imaginario social en relación a los migrantes venezolanos y a su realidad. Es 
importante conocer si los medios cumplen con su misión informativa y comunicativa o van más allá, difundiendo hechos que pueden ocasionar la creación de estereotipos negativos en torno a la población venezolana.

La interrogante que guía la elaboración del presente proyecto de investigación es la siguiente: ¿cómo se construyó el discurso periodístico del diario La Nación de la ciudad de Tulcán en relación al proceso migratorio de la población venezolana hacia Ecuador, durante los meses de enero a marzo de 2019, y de qué manera esto influyó en la creación de representaciones sociales?

A fin de encontrar una respuesta para esta pregunta, se definieron los siguientes objetivos específicos: enunciar los principales elementos que generan la salida de ciudadanos venezolanos hacia Ecuador, identificar las representaciones sociales presentes en el discurso periodístico del diario La Nación de Tulcán sobre el proceso migratorio venezolano, y formular las líneas base para la creación de un protocolo que permita combatir estereotipos xenófobos en la producción informativa.

La metodología del estudio se centra en la aplicación de la versión técnica comunicacional -VTC- (Massoni 2013a), para profundizar en la problemática, el contexto y los actores alrededor de diario La Nación, y su manejo del tema migratorio. También se toman en cuenta los principios del análisis crítico del discurso (Van Dijk 1999), a fin de identificar las representaciones sociales presentes en el discurso periodístico relacionado con el tema migratorio. En total se analizaron cuatro notas informativas.

\section{Migración venezolana}

La movilidad se entiende como un proceso humano, la expresión del cumplimiento de un derecho, motivado por diferentes causas. Presenta la clara intención de permanecer en un territorio diferente a aquel en el cual una persona o grupo de personas se encuentra en la actualidad. Mas allá de las implicancias sociales, económicas, políticas y culturales que se insertan en el tema de la movilización humana, según menciona Llamas, esta debe ser comprendida como un derecho humano:

El mismo se encuentra reconocido en los principales instrumentos regionales y universales de esa materia, así como en un amplio número de constituciones y leyes nacionales. Es un derecho humano universal, interdependiente e indivisible como lo son el derecho a la vida, a la propiedad privada, a la salud, la libertad de expresión o cualquiera del mismo orden. $(2016,147-8)$

Desde esta perspectiva, la movilidad se configura como un derecho que otorga a las personas la posibilidad de transitar libremente alrededor del mundo. Es un proceso que da cuenta de las múltiples realidades que las personas atraviesan. Consiste en cruzar las fronteras políticas y geográficas tanto determinadas y reconocidas por las legislaciones nacionales e internacionales, como formuladas y adoptadas por los diferentes países (Ortiz 2018). Al 
ser un derecho esencial de las personas, es responsabilidad de los Estados y su institucionalidad asegurar que este proceso se realice bajo condiciones de justicia y dignidad.

Durante la mitad del siglo XX, América Latina fue testigo de tres procesos migratorios que tuvieron como destino el continente europeo. Las razones que provocaron esta situación fueron la fuerte inestabilidad económica y política, la ausencia de fuentes de trabajo y el aumento acelerado de la violencia y la inseguridad. En consonancia con esta afirmación, Vásquez, Castillo y Lera (2015) refieren que la población migrante atraviesa altos niveles de vulnerabilidad, que la obligan a salir de su lugar de origen para tratar de encontrar nuevas oportunidades y medios de subsistencia.

En este contexto, la migración forzada a la que se enfrentan los ciudadanos venezolanos da cuenta de la grave crisis que atraviesan ese país en las dimensiones política, social y económica, además de la ausencia total de programas u opciones para solucionarla (Díaz 2016, 58). La información expuesta en la Encuesta Nacional de Condiciones de Vida (ENCOVI) indica que los niveles de pobreza en Venezuela se incrementaron del 48,4 $\%$ en 2014 al 87 \% en 2017; de este porcentaje, el 61,2 \% traspasa los límites de la pobreza extrema (Bermúdez et al. 2018).

Los datos muestran que la migración forzada de los ciudadanos venezolanos hacia otras naciones sudamericanas es consecuencia de una grave crisis institucional y estatal que no muestra interés por la creación de políticas y programas sociales que beneficien a su población. Ante esta realidad, los habitantes no sienten que cuentan con las garantías suficientes para vivir en condiciones de equidad, seguridad y justicia.

Ecuador, en este contexto, se ha convertido en un país receptor para quienes deciden asentarse de manera indefinida en el territorio, al tiempo que opera como una zona de tránsito para quienes tienen la intención de llegar hacia otras naciones dentro de las fronteras latinoamericanas. Izurieta (2018) menciona que algunos de los factores que influyen son la dolarización, una mayor seguridad, la estabilidad, la cercanía, el idioma, la accesibilidad de la normativa migratoria y la posibilidad de mejorar sus condiciones de vida.

Pese a estos elementos que los ciudadanos venezolanos ven como beneficiosos, la realidad es que Ecuador abriga problemáticas propias que se profundizan con la llegada masiva de personas extranjeras, debido a la ausencia de una institucionalidad con la capacidad para enfrentar esta problemática social. Sumado a esto, las dificultades de carácter económico y social - como la falta de oportunidades laborales, el precario funcionamiento de los sistemas de salud y educativo, entre otros - complejizan aún más la realidad del país en torno a la migración masiva que experimenta en la actualidad.

\section{El discurso periodístico y la construcción de realidades}

El discurso se concibe como una expresión de las dimensiones relacionadas con la idea de poder, entendiéndola en la integralidad de su significado, es decir, operando desde 
diferentes escenarios para legitimar aquello que se considera normal en la sociedad. Es por medio del uso de la palabra, de su enfoque e intencionalidad, que se instauran visiones sobre los hechos que suceden dentro de los límites de la estructura social. Sobre esto, Calsamiglia y Tusón manifiestan:

Hablar de discurso es, ante todo, hablar de una práctica social, de una forma de acción entre las personas que se articula a partir del uso lingüístico contextualizado, ya sea oral o escrito. El discurso es parte de la vida social y a la vez un instrumento que crea la vida social. Desde el punto de vista del discurso, hablar o escribir no es otra cosa que construir piezas textuales orientadas a unos fines y que se dan en interdependencia con el contexto (lingüístico, local, cognitivo y sociocultural). $(2001,15)$

Siguiendo las ideas propuestas por Calsamiglia y Tusón, se afirma que el discurso opera por la intervención y el uso de los distintos elementos lingüísticos, construyendo simbolismos, significados y representaciones, elementos que hacen posible el funcionamiento de imaginarios y representaciones que el poder considera importantes. Al ser el lenguaje un elemento versátil y adaptable, será manejado dependiendo de la intencionalidad del mensaje que se quiera dar a conocer.

El discurso, mediante el lenguaje y sus múltiples connotaciones, crea formas particulares de entender, asimilar y analizar los hechos. Las construcciones discursivas son el reflejo de la ideología de un individuo o grupo. Tal como señala Van Dijk (2006, 245), la manera en que se aborda un contexto depende directamente del enfoque desde el cual se expresa, y puede tener como finalidad ejercer cierta influencia sobre la opinión pública.

En este marco, el discurso funciona a manera de metáfora: "Las metáforas ontológicas más obvias son aquellas en las que el objeto físico se especifica como una persona. Esto nos permite comprender una amplia diversidad de experiencias con entidades no humanas en términos de motivaciones, características y actividades humanas" (Lakoff y Johnson 2015, 71). Es así que la construcción del escenario social se configura como el reflejo de los intereses de los individuos.

Por lo tanto, el discurso cuenta con elementos que influencian los modos de comprender los hechos y acontecimientos del mundo; la manera en que sea estructurado se reflejará en las ideas, creencias, actitudes y comportamientos de los sujetos sociales. De esta manera, quien tiene el poder sobre el discurso termina influenciando los procesos que se desarrollan en el entorno social. Aquí se manejan emociones e intereses particulares asociados con la defensa del statu quo.

Tal como señala Foucault (2005), es en el discurso donde se establece el límite entre lo real y lo imaginario; con base en estos criterios se crean imaginarios cuya función es garantizar que se mantenga el aparente equilibrio social. Las representaciones implantadas desde las producciones discursivas definen la visión, el significado y el sentido de las problemáticas sociales. 
Los medios de comunicación tienen la facultad de relatar y crear realidades que son mostradas al mundo como parte del contexto en el cual habitan. Cada sociedad crea su propia percepción teniendo como base el discurso presentado desde los mass media. Es a través de ellos que se construyen las ideologías que definen las percepciones de la sociedad frente a hechos determinados. Sin embargo, esta configuración de la realidad responde a una línea editorial y a unos intereses específicos que muestran versiones parcializadas y poco claras de problemáticas sociales como la migración.

\section{Las representaciones sociales como elemento del hecho noticioso}

Las representaciones sociales se definen como "el punto de unión entre las realidades discursivas (comunicativas) y fácticas, entre la imaginación y la acción, actuando como nexo entre el universo interior y el exterior de los sujetos" (Rubira y Puebla 2018, 148). Es decir, sirven como mecanismos a través de los cuales se pueden interpretar el mundo y las diferentes visiones que en él convergen. Tal como plantean Berger y Luckmann (1986), las representaciones construyen el objeto al cual hacen referencia, y en consecuencia tienen el poder de construir contextos sociales con base en la interpretación que los seres sociales hacen de su entorno.

Las representaciones sociales son entidades simbólicas cuyo significado es compartido socialmente y actúan como condicionantes de la acción. Están vinculadas con las ideologías, nichos de poder y frentes de resistencia. El carácter transversal de las representaciones las vincula con el discurso. Según Fairclough están atadas a las prácticas sociales y se instalan mediante el uso del lenguaje, de allí que la realidad sea vista como la acumulación de significados que cobran sentido en un determinado contexto (Cajas, Casimiro y Pasquel 2017, 95)

$\mathrm{Al}$ estar vinculado con el lenguaje y sus códigos, el discurso periodístico dentro del ejercicio comunicativo tiene el poder de crear representaciones sociales en coyunturas particulares. Por lo tanto, se trata de constructos simbólicos con significados compartidos a nivel social, que determinan los marcos de actuación de un grupo específico frente a circunstancias concretas. Se encuentran directamente relacionadas con la actividad discursiva y se fortalecen en los procesos que tienen lugar en el ámbito de la comunicación por medio de los hechos noticiosos que se transmiten.

Siguiendo esta idea, destaca lo señalado por Ponte (2014), quien afirma que las representaciones sociales son la suma de datos, puntos de vista, formas de comportamiento y creencias que se dan en relación a un hecho u objeto determinado. Se entiende entonces que las representaciones sociales son el conjunto de ideas y sensaciones que un grupo tiene frente a algo o alguien. Se trata de una manera de construir e interpretar realidades, tomando como ejes de referencia el sistema de valores, las creencias y los conocimientos que existen sobre un tema específico. 
Así, las representaciones creadas a partir de los hechos noticiosos generan simbolismos y significados que determinan las formas de actuar de un conglomerado respecto a una realidad concreta. Estas realidades adquieren la categoría de verdaderas, con lo que generan formas de reaccionar por parte de la sociedad, y se ven reflejadas en los actos que los sujetos sociales realizan en su cotidianidad.

A propósito de esto, Verón (1981) señala que la actualidad, en tanto se configura como la realidad social, existe gracias a los medios de comunicación. Esto significa que los hechos como tal no existen si antes no han sido construidos por los mass media. Una vez que han sido producidos y dados a conocer, provocan diferentes efectos que los terminan convirtiendo en acontecimientos sociales de amplia relevancia, al tiempo que se reproducen y cobran significado por el contexto y el punto de vista desde el cual son leídos.

Desde esta perspectiva, la construcción del acontecimiento se enfrasca en tres dimensiones: la semiótica, que hace referencia a la producción del hecho noticioso; la ideológica, en tanto da cuenta de la existencia de un problema; y la del poder, que procura posicionar mensajes para convertirlos en representaciones sociales que se reproducen en el imaginario de la sociedad y pasan a tener significados que se reflejan en actores particulares.

Ante este planteamiento, Charaudeau señala que "los medios quieren dar cuenta de la realidad y en realidad la están construyendo [...]. La base del funcionamiento de los medios en la sociedad es una relación triangular, entre la realidad, los medios y el pueblo" (en García 2012, 309). En este sentido, el discurso periodístico creado desde los mass media no cumple la función de ser una representación exacta de la realidad, sino que está atravesada por una serie de filtros e intereses que terminan creando imágenes simplificadas del acontecimiento a fin de llegar a la mayor cantidad de público posible. Para esto, hacen uso de estereotipos con significados compartidos dentro de un grupo social.

Así, en la construcción del hecho noticioso desde los medios de comunicación, según Charaudeau, el público se posiciona como observador a distancia del espectáculo que se le muestra, para simplemente advertirlo y juzgarlo, o bien se "proyecta en los dramas del mundo y, al mismo tiempo, permanece a distancia de su espectáculo; puede sentir las alegrías y los sufrimientos del mundo y al mismo tiempo, juzgarlos" (2005, 328).

Por lo tanto, la construcción del acontecimiento, desde los medios de comunicación, se convierte en un trabajo que procura mostrar la realidad apelando a los sentidos, las emociones y las sensaciones, generando así la espectacularización de los hechos con el uso de términos y frases con significados propios dentro de una determinada sociedad. En este contexto, el ciudadano pasa, de ser un sujeto que requiere informarse, a ser un consumidor al que se atrapa por medio de un discurso que apela a su emotividad y con el cual termina relacionándose tanto para identificarse como para mostrarse contrario. Esto último sucede principalmente con sucesos que alteran la cotidianidad y dan paso a la conformación de representaciones sociales.

Con base en estos planteamientos se establece que los discursos periodísticos están atravesados por representaciones sociales construidas por medio de códigos, mensajes, ideas 
e imágenes que se socializan a través de la difusión de los medios de comunicación. Los discursos son decodificados, asimilados y entendidos, y dan paso entonces a la conformación de conductas consensuadas que se legitiman asumiéndose como verdades absolutas. "El objetivo de toda representación social encarnada en determinado grupo social es argumentar y convencer, a propios y extraños, de que su representación es algo objetivo y evidente" (Ponte 2014, 113).

Es la prensa la que valida, por medio de su discurso, estas formas de ver, entender y actuar en esa realidad social que se reconoce y acepta como única. Estas representaciones adquieren aún más significado cuando dentro de una realidad se presentan fricciones; es entonces cuando los medios de comunicación transmiten, junto con los hechos noticiosos, imaginarios que se posicionan en los mensajes.

Entender la migración desde una mirada comunicacional implica enmarcarla dentro de los relatos creados desde los medios de comunicación, los cuales asumen la responsabilidad de dar a conocer el desarrollo de los acontecimientos que suceden en la sociedad. Se debe considerar que, en los últimos tiempos, el tema migratorio ha sido una de las problemáticas que en mayor medida se ha visto reflejada en los relatos periodísticos.

A propósito, Ruiz-Aranguren (2018) plantea que en la construcción de la información que se transmite a través de los mass media existe un alto grado de incidencia de actores políticos, sociales e institucionales, además de que falta una estrategia clara que direccione la manera en que se debe tratar la migración. La autora afirma que el relato periodístico influye en la construcción de estereotipos en torno a las poblaciones migrantes, ya que se les atribuye la responsabilidad de problemáticas sociales relacionadas con la violencia o la delincuencia.

Según Igartua et al. (2007), tomando en cuenta la teoría del framing, esto ocurre sobre todo en los espacios televisivos, mientras que en la prensa se observa una mayor diversidad de enfoques políticos, sociales y culturales, entre otros. Al tener los medios televisivos un mayor alcance, sin embargo, contribuyen a la construcción y perpetuación de estereotipos negativos en las audiencias respecto a las poblaciones migrantes, situación que de cierta manera termina normalizando, por ejemplo, la discriminación o la exclusión.

La realidad como tal está mediada por una construcción discursiva que crea representaciones sociales que se van implantando como realidades compartidas por gran parte de los miembros de un contexto determinado; en algunos casos incluso alcanzan el grado de verdades absolutas. Son reproducidas de tal manera que no permiten otra interpretación del acontecimiento.

Lo mismo sucede con la organización de las representaciones sociales más generales. Así, si los refugiados son caracterizados en el discurso político o en un editorial de periódico en términos esencialmente socioeconómicos, y por tanto como impostores, como gente que solo viene aquí para vivir a costa de nuestro bienestar, entonces una opinión genérica como esa puede también definir la representación social (el esquema de grupo) que la gente construye (o confirma) sobre ellos. (Van Dijk 1999, 31) 
El relato periodístico creado por los medios de comunicación en torno al tema migratorio tiene un alto grado de incidencia en la creación de realidades. Las ideas, las palabras y los enfoques que difunden los medios de comunicación provocan en la sociedad la adopción de representaciones sociales que alcanzan el grado de verdad. Dentro de este estudio, se toman en cuenta los elementos desarrollados en este apartado para analizar la manera en que el diario La Nación relata en su discurso periodístico el tema migratorio.

\section{La versión técnica comunicacional (VTC) aplicada al análisis de diario La Nación}

El diario La Nación circula en la provincia del Carchi los días domingo con un tiraje de 3600 ejemplares. Dentro de sus principios destaca el hecho de que se reconoce como una empresa de patrimonio familiar que busca la rentabilidad suficiente para asegurar el liderazgo sostenido como un medio de comunicación referente dentro del contexto regional. Forma parte de una empresa de carácter privado, cuyas lógicas de producción noticiosas se centran en asegurar la rentabilidad de su negocio, brindando información de actualidad que interese a su público objetivo en función de la coyuntura de la frontera norte del país.

La comunicación estratégica "confronta el trabajo de campo de la investigación con las versiones de las matrices socioculturales vinculadas a la problemática" (Massoni 2013a, 28), lo que metodológicamente desde la Escuela de Rosario se denomina versión técnica comunicacional (VTC), entendida como un diálogo de saberes en el que se realiza un reconocimiento de la realidad del objeto de estudio.

Este proceso tiene el fin de lograr una visión integral de la realidad de estudio para plantear estrategias que den respuestas eficaces a las problemáticas detectadas. Teniendo en cuenta la naturaleza del análisis que se plantea, cabe mencionar que los medios de comunicación han realizado el seguimiento del tema migratorio por medio de diferentes soportes; por esta razón, entender los hechos sociales y comprender la realidad tal como se desarrolla hoy en día implica reformular las perspectivas tradicionales respecto a los procesos de recepción.

Repensar el tema migratorio en los medios de comunicación requiere comprender las diferentes dinámicas que se desarrollan en los niveles políticos, sociales, económicos y de derechos humanos. No puede ser visto y analizado únicamente como un hecho a partir del cual las personas decidieron abandonar su país sin tomar en cuenta los contextos. Es fundamental asumir la realidad como un proceso no terminado, en constante transformación y cambio.

En consonancia con el tema de la complejidad del tema migratorio, aparece la investigación enactiva en comunicación (IEC) como un punto de encuentro con la diversidad. Todos los saberes y puntos de vista son considerados, lo que hace de lo complejo un espacio fluido que da cuenta de la transformación de la realidad por medio de la aplicación de la comunicación estratégica. Según explica Massoni (2016), es necesario desplegar la comuni- 
cación, descomprimirla, abordar su multidimensionalidad sin descartar su fluidez; en definitiva, ubicarse dentro de las problemáticas sociales como comunicadores estratégicos. A decir de Massoni, se trata de

una propuesta metodológica que se hace a partir de una fuerte autocrítica respecto de las modalidades tradicionales de pensar y de hacer investigación en comunicación. Trabaja en rebasar algunos de los reduccionismos de las teorías clásicas y para eso se basa en un sistema explicativo que redefine a la comunicación como encuentro en la diversidad. (2018, 46-47)

Como explica la autora, esta teoría se fundamenta en las epistemologías del Sur, cuyos antecedentes son el pensamiento comunicacional latinoamericano, la filosofía del buen vivir de los pueblos originarios, y los nuevos paradigmas de la ciencia, relacionados con la complejidad, la fractalidad y lo fluido. Por lo tanto, enactuar significa plantear estrategias de comunicación desde la multidimensionalidad para lograr un cambio social conversacional, como un aporte que permite emerger al encuentro de la diversidad.

En el marco de este estudio, la comunicación estratégica se define como la suma de acciones que procuran la generación de sentido y significación entre el mensaje que se genera, los receptores y las interpretaciones que estos dan a la información que reciben. Desde la perspectiva de Massoni, Piola y Bussi (2017), se hace referencia a un cambio social conversacional que toma en cuenta a todos los actores que forman parte de la problemática que se analiza, y para la cual se buscan posibles soluciones.

La VTC y la comunicación enactiva como tal se presentan como herramientas que permiten abordar los problemas comunicacionales desde un enfoque integral y fluido (Massoni 2016). Se acercan a los diferentes actores, reconociéndolos como parte fundamental de las dinámicas, los escenarios, las relaciones y demás elementos que forman parte de las realidades emergentes resultantes de los procesos comunicativos. Es por esta razón que sus fundamentos, principios y herramientas fueron utilizados, dentro de este estudio, para la elaboración del análisis contextual del diario La Nación.

A fin de conocer mejor la problemática, el contexto y los actores alrededor de $L a$ Nación y su manejo del tema migratorio, a continuación se presenta una serie de instrumentos de la VTC. Se trata de diferentes matrices en las que se detallan los siguientes aspectos: frase núcleo del problema, componentes y aspectos del problema, niveles del problema, reconocimiento de actores, reconocimiento de matrices socioculturales y caracterización de matrices socioculturales.

Siguiendo los planteamientos de Massoni (2013b), en la Tabla 1 se presenta de manera concisa el problema a analizar en el estudio, determinado que el relato periodístico tiende a presentar una mirada estereotipada de los hechos situados alrededor del fenómeno migratorio. 
Tabla 1

Frase núcleo del problema

\begin{tabular}{|l|l|}
\hline \multicolumn{2}{|l|}{ Versión técnica comunicacional (VTC) del proyecto IEC Migración y Comunicación } \\
\hline Tema & Comunicación y migración \\
\hline Para quién & Diario La Nación (Tulcán) \\
\hline $\begin{array}{l}\text { Frase núcleo } \\
\text { de la VTC }\end{array}$ & $\begin{array}{l}\text { Visión estereotipada de las representaciones sociales de la población } \\
\text { venezolana migrante en el discurso mediático en prensa escrita. }\end{array}$ \\
\hline
\end{tabular}

Fuente: Massoni (2013b)

Elaboración propia

Siguiendo los principios de la VTC se identifican los componentes que forman parte del problema, entendiéndolos como los aspectos diferenciadores que constituyen la multidimensionalidad de la realidad en estudio. En este caso específico se presta atención al componente comunicacional.

Tabla 2

Niveles del problema: Componente comunicacional

\begin{tabular}{|l|ll|}
\hline \multicolumn{2}{|l|}{ Versión técnica comunicacional (VTC) del proyecto IEC Migración y Comunicación } \\
\hline \multirow{3}{*}{ Síntomas } & 1. & Parcialización de la información \\
& 2. & Visión estereotipada de la imagen de los ciudadanos venezolanos \\
& 3. & Tratamiento informativo xenófobo \\
\hline Consecuencias & 1. & Escaso conocimiento de la realidad migratoria venezolana \\
& 2. & Rechazo hacia los ciudadanos venezolanos \\
& 3. & Inadecuado tratamiento informativo \\
\hline Aspecto & 1. & Los medios de comunicación se rigen a la agenda política \\
Comunicacional & 2. & $\begin{array}{l}\text { Prevalencia de hechos noticiosos negativos relacionados a los } \\
\text { migrantes venezolanos }\end{array}$ \\
& 3. & $\begin{array}{l}\text { Inexistencia de estrategias comunicacionales para abordar el tema } \\
\text { migratorio }\end{array}$ \\
\hline Causas próximas & 1. & $\begin{array}{l}\text { Históricamente los medios de comunicación han respondido a } \\
\text { poderes políticos y económicos }\end{array}$ \\
& 2. & $\begin{array}{l}\text { Prevalencia, en los últimos tiempos, de un tratamiento amarillista de } \\
\text { la información }\end{array}$ \\
& 3. & $\begin{array}{l}\text { Falta de interés en generar protocolos de tratamiento informativo } \\
\text { para temas migratorios }\end{array}$ \\
\hline Causas básicas & 1. & $\begin{array}{l}\text { Defensa de intereses de dueños de medios de comunicación } \\
\text { 2. Sensacionalismo: la información como un producto de venta }\end{array}$ \\
& 3. & Estructura organizacional tradicional \\
\hline
\end{tabular}

Fuente: Massoni (2013b)

Elaboración propia 
En relación al componente comunicacional, el principal síntoma es la parcialización de la información; la consecuencia de esto es el escaso conocimiento de la realidad migratoria venezolana; la causa próxima da cuenta de que históricamente los medios de comunicación han respondido a poderes políticos y económicos; y la causa básica es la defensa de los intereses de los dueños de medios de comunicación.

En la Tabla 3 se reconoce a los actores vinculados al problema en el componente comunicacional. Su definición permite conocer su nivel de incidencia en la problemática, para una mejor comprensión de los hechos referentes a la visión estereotipada de las representaciones sociales de la población venezolana migrante en el discurso mediático durante los meses de enero a marzo de 2019.

Tabla 3

Reconocimiento de actores del componente comunicacional

\begin{tabular}{|l|c|}
\hline \multicolumn{2}{|c|}{ Versión técnica comunicacional (VTC) del proyecto IEC } \\
Migración y Comunicación
\end{tabular}

Fuente: Massoni (2013b)

Elaboración propia

La aplicación de la VTC, para conocer la realidad del diario La Nación en torno a las representaciones sociales en el discurso periodístico, permitió identificar los diferentes elementos que inciden en esta problemática. Por medio de su aplicación fue posible asumir el tema de migración como una realidad compleja en la que intervienen diferentes actores y escenarios que, al interactuar, dan paso a la creación de nuevas dimensiones sobre las cuales es necesario indagar para lograr una verdadera transformación en la manera de informar.

En las matrices relacionadas al componente comunicacional se determinaron como relevantes los siguientes aspectos: los medios de comunicación se rigen a la agenda política, prevalecen hechos noticiosos negativos relacionados a los migrantes venezolanos, y faltan estrategias comunicacionales para abordar el tema migratorio.

\section{Análisis del discurso de las notas periodísticas}

Para analizar las representaciones sociales que se manejan en las publicaciones del diario La Nación en relación al tema migratorio, se toman en consideración algunas de las categorías de análisis crítico del discurso propuestas por Teun Van Dijk (1999). Es importante señalar que durante los meses de enero a marzo de 2019 se realizó un seguimiento de 
los artículos publicados por este medio de comunicación en su edición impresa. Dentro de este estudio se hace referencia a cuatro, tal como se detalla en la siguiente tabla:

Tabla 4

\section{Artículos del diario La Nación}

\begin{tabular}{|l|l|}
\hline Fecha & Página / Titular \\
\hline $\begin{array}{l}\text { Domingo/Lunes } \\
\text { 13/14 de enero de 2019 }\end{array}$ & $\begin{array}{l}\text { Portada } \\
\text { En el centro de Tulcán adolescente venezolano apuñala a joven }\end{array}$ \\
\hline $\begin{array}{l}\text { Domingo/Lunes } \\
\text { 13/14 de enero de 2019 }\end{array}$ & $\begin{array}{l}\text { Pág. } 7 \\
\text { Adolescente venezolano apuñala a joven en el centro de Tulcán }\end{array}$ \\
\hline $\begin{array}{l}\text { Domingo/Lunes } \\
\text { 27/28 de enero de 2019 }\end{array}$ & $\begin{array}{l}\text { Pág. } 6 \\
\text { Más de 2000 venezolanos dejan la terminal de Tulcán }\end{array}$ \\
\hline $\begin{array}{l}\text { Domingo/Lunes } \\
\text { 27/28 de enero de 2019 }\end{array}$ & $\begin{array}{l}\text { Pág. 7 } \\
\text { Venezolanos en desacuerdo con puesta en marcha del pasado judicial }\end{array}$ \\
\hline
\end{tabular}

Fuente: La Nación

Elaboración propia

En este apartado es necesario aclarar que las fechas de las publicaciones corresponden a dos días, ya que es la manera en que La Nación coloca esta información en su edición impresa. En la página de portada del domingo/lunes 13/14 de enero de 2019, se muestra el siguiente titular: "En el centro de Tulcán adolescente venezolano apuñala a joven". Quien accede a este contenido inmediatamente empieza a crearse una imagen de lo sucedido, incluso sin haber leído la nota.

En la ampliación de la página 7, titulada "Adolescente venezolano apuñala a joven en el centro de Tulcán", además de narrar de manera cronológica los hechos, se aprecia una contradicción importante: si bien el titular y algunos datos de la nota aseguran que este delito fue cometido por un ciudadano venezolano, en el mismo relato se lo reconoce únicamente como presunto responsable. Además, cabe mencionar que existe un interés particular por destacar el dato acerca de la nacionalidad de quien es considerado el agresor. Así, se continúa perpetuando la idea de que tener una determinada ciudadanía va de la mano del cometimiento de crímenes.

Algo similar se observa en la nota del domingo/lunes 27/28 de enero de 2019 "Más de 2000 venezolanos dejan la terminal de Tulcán": de manera reiterativa se hace referencia a la nacionalidad de las personas. A pesar de que el antetítulo menciona el temor y el pánico xenofóbico, este tema no se desarrolla ni se presenta información relacionada a la manera en que se manifiesta o se percibe por parte de la población.

Como resultado de un hecho específico acontecido en la ciudad de Ibarra durante el mes de enero, en los medios de comunicación se creó un discurso en el cual se apuntaba a los ciudadanos de origen venezolano como el mayor problema que la sociedad ecuato- 
riana debía enfrentar para lograr la paz y el equilibrio social. Este mensaje se reforzó con las declaraciones realizadas desde el Gobierno central en la voz del presidente Lenín Moreno, quien en sus redes sociales publicó un mensaje refiriéndose a este acontecimiento, que generó conmoción en todo el país.

En cierta manera, durante este tiempo los medios de comunicación y el Gobierno colocaron en el escenario el tema de la migración venezolana como una problemática que terminaba afectando la estabilidad, la seguridad y el bienestar de todos los ciudadanos ecuatorianos. Se crearon representaciones sociales que dieron mayor fuerza a ideas preconcebidas acerca de las personas extranjeras, que, como consecuencia de la situación de su país, se vieron obligados a salir hacia otras naciones en busca de nuevas oportunidades de vida.

La declaratoria del presidente Lenín Moreno dio paso a la firma del Acuerdo Ministerial 001, en el que se exige como requisito para ingresar a Ecuador que los ciudadanos venezolanos cuenten con el pasado judicial apostillado. Esta situación vulnera su derecho a la libre movilidad, tomando en cuenta la realidad en la que se encuentra Venezuela y la dificultad de realizar cualquier tipo de trámites en ese país, así como el tiempo que se tardaría en obtener este documento.

Además, tampoco se tomó en cuenta a las personas que habían salido de sus lugares de origen antes de que se emitiera esta normativa. En la nota publicada el 27/28 de enero de 2019, titulada "Venezolanos en desacuerdo con puesta en marcha del pasado judicial", se relata esta información, y se resaltan la inconformidad y las exigencias que realizan al Gobierno de Ecuador para no cumplir con esta medida.

En el análisis de los artículos se hace evidente el uso de estrategias de carácter cognitivo, que dan significado al relato discursivo otorgándole elementos de veracidad sobre el hecho que se difunde. Las intenciones pueden estar enfocadas en cumplir el rol de informar, al ser un medio de comunicación importante en la ciudad de Tulcán. El propósito es mostrar la realidad de migración venezolana desde las problemáticas que esto genera a nivel social, principalmente en lo relacionado al tema de la seguridad.

Se genera así una producción discursiva enfocada en retratar acontecimientos que ponen en el escenario principalmente problemas de tipo social, pero no se profundiza en los hechos políticos y económicos que han provocado la masiva salida de ciudadanos desde su país de origen. Es decir, se cuenta una realidad fragmentada que centra únicamente la atención en la espectacularización de los hechos de violencia, conmoción, inseguridad y drama.

La creación del discurso periodístico en torno a la migración requiere la comprensión de las diferentes dimensiones y dinámicas que en ella se desarrollan. Los procesos migratorios, en el relato periodístico, deben ser abordados y entendidos en su verdadera complejidad; de esta manera se evitaría la formación de representaciones sociales relacionadas con hechos negativos como la delincuencia y la violencia, entre otros, que terminan 
provocando en la sociedad el aparecimiento de conductas como la xenofobia y el racismo, que agravan y vulneran los derechos y la estabilidad tanto de la población migrante como de la receptora.

El discurso periodístico tiene un gran alcance y poder. La manera en la cual se crea, organiza y difunde termina definiendo aquello de lo se debe hablar y cómo hacerlo. Es su agenda la que define los acontecimientos a informar, mostrando un relato de la realidad y dando paso a la conformación de representaciones sociales que se implantan en el imaginario de las personas. Siguiendo a Van Dijk (2006), las realidades relatadas desde los medios de comunicación terminan configurando representaciones sociales que se instalan en el imaginario de la colectividad.

El poder juega de diferentes maneras y no se mantiene estático; en este contexto los mass media cumplen un rol fundamental. Al respecto, Foucault (2005) señala que el discurso aspira a lograr ciertos fines: si bien puede ser considerado un instrumento de lucha, también es utilizado para hacer que la sociedad actúe de cierta manera en función de las realidades a las cuales se enfrenta, y llega incluso a ejercer un tipo de control.

Así, el hecho de remarcar, repetir e incluso maximizar los acontecimientos negativos en las notas que abordan la migración venezolana incide en la creación de representaciones asociadas a problemáticas de violencia, inseguridad y conflictividad. En cierta medida se enmarca a todo ciudadano venezolano que se encuentre en el país en una estructura negativa que se refuerza constantemente con los discursos creados y transmitidos por los diferentes medios de comunicación; en este estudio específico, el diario La Nación, que circula en la ciudad de Tulcán con un tiraje de 3600 ejemplares y con una versión online a la cual pueden acceder otras personas desde distintos lugares.

Las notas informativas analizadas ayudaron a determinar que la línea editorial del diario La Nación apunta a la repetición de un discurso referente al tema migratorio, en el cual se señalan constantemente aspectos asociados a la inseguridad, la violencia y la conflictividad. Se difunde de manera reiterativa la idea del miedo frente al extranjero, lo que da paso a la instauración de representaciones xenófobas que intentan colocar toda la responsabilidad de la situación actual de la ciudad de Tulcán en estos actores que forman parte de la nueva cotidianidad. Cabe mencionar que esto, además, es una estrategia que trata este tema desde las lógicas del espectáculo, para captar la atención y el interés del público.

De cierta manera, desde el diario La Nación se da un proceso de ocultamiento y desconocimiento de la falta de atención histórica por parte del Estado y el resto de la institucionalidad a la toma de acciones que mejoren las condiciones de vida de la población de esta parte del país. No es una realidad ajena que las zonas fronterizas atraviesan serias condiciones socioeconómicas y esto acarrea problemáticas que no son nuevas; sin embargo, han encontrado en los migrantes venezolanos un nuevo responsable. 


\section{Conclusiones}

La migración en América Latina tiene un fuerte vínculo con dimensiones sociales que afectan la estabilidad de la población. La inestabilidad política, la desigualdad social y el constante crecimiento de la pobreza - así como las consecuencias derivadas de esto, como la violencia y la xenofobia, además de la falta de una solución a estas problemáticas por parte de las autoridades competentes - han generado que las personas se vean forzadas a abandonar sus naciones de origen.

Los principales elementos que han ocasionado la salida de ciudadanos venezolanos hacia Ecuador son la inestabilidad política, deficientes condiciones socioeconómicas, el aumento creciente de los niveles de inseguridad y violencia, la escasez de productos de primera necesidad como alimentos y medicina, el crecimiento inflacionario y una constante reducción del valor de su moneda. Todo esto ha provocado que no encuentren en su país los medios suficientes para sobrevivir, por lo que se ven forzados a trasladarse a otras naciones dentro de América Latina. Sin embargo, no se puede desconocer el hecho de que los países de Sudamérica atraviesan condiciones similares en lo referente a sus condiciones de vida y a su situación social, política y económica.

Al ser la migración un tema de interés general, ha cobrado relevancia dentro de los medios de comunicación, los principales voceros de este hecho que involucra al conjunto de la sociedad en sus múltiples dimensiones. En el caso del diario La Nación y su producción discursiva en torno al tema migratorio, se observan elementos que corroboran que el enfoque que asumen frente a un tema determina la forma en que este es comprendido por la sociedad.

Las notas informativas sobre la migración venezolana publicadas por diario $\mathrm{La} \mathrm{Na}$ ción tienen como principales temáticas los problemas que se derivan de esta situación para la ciudad de Tulcán, particularmente con lo relacionado a la seguridad. La producción discursiva de este medio de comunicación crea relatos alrededor de la violencia, la delincuencia y el incremento de los niveles de inseguridad y conflictividad, los cuales se van posicionando en el imaginario de las personas para dar paso a la conformación de representaciones sociales que colocan como los principales actores de esta situación a los ciudadanos venezolanos.

Las estructuras negativas creadas sobre los migrantes venezolanos se refuerzan constantemente con la producción discursiva difundida desde el medio de comunicación. La repetición de los hechos negativos y la referencia a la ciudadanía van configurando en la población local un miedo y un rechazo que derivan en actitudes xenófobas que atentan contra los derechos y la seguridad de estas personas.

El uso reiterativo, dentro del discurso periodístico, de términos como extranjero, venezolano, violencia y delincuencia hace que la opinión pública configure representaciones sociales con connotaciones negativas, colocando a todos los migrantes en una misma 
categoría. En este contexto, y tras el análisis realizado, se reafirma la importancia y la responsabilidad que tienen los medios de comunicación de informar desde un enfoque de respeto a los derechos humanos, sobre todo cuando se relatan realidades tan complejas, diversas y dinámicas como la migración.

\section{Referencias}

Berger, Peter, y Thomas Luckmann. 1986. La construcción social de la realidad. Buenos Aires: Amorrortu.

Bermúdez, Yovanny, Rina Mazuera, Neida Albornoz, y Miguel Morffe. 2018. Informe sobre la movilidad humana venezolana. Realidades y perspectivas de quienes emigran. Lima: CEPAL.

Cajas, Verónica, Consuelo Casimiro, y Lorenzo Pasquel. 2017. "Las representaciones sociales y el discurso noticioso de la Ley Universitaria - Perú”. Comunic@cción 8 (2): 94-104. https://bit.ly/3fmifMC.

Calsamiglia, Helena, y Amparo Tusón. 2001. Las cosas del decir. Manual de análisis del discurso. Barcelona: Ariel.

Charaudeau, Patrick. 2005. “¿Nos manipulan los medios?”. CIC. Cuadernos de Información y Comunicación 10: 319-30. https://bit.ly/3brwnTK.

Díaz, Daniela. 2016. "Principio de igualdad y no discriminación de los derechos humanos de las mujeres inmigrantes venezolanas profesionales en Quito, Ecuador, 2014-2015”. Tesis de maestría, Universidad Andina Simón Bolívar, Sede Ecuador.

Foucault, Michel. 2005. El orden del discurso. Buenos Aires: Tusquets.

García, Kevin. 2012. "Un gusano en la manzana informativa: Entrevista con Patrick Charaudeau”. Revista Nexus Comunicación 1 (11): 308-13. https://bit.ly/3yaBh1k.

Igartua, J. J., C. Muñiz, J. A. Otero y M. de la Fuente. 2007. “El tratamiento informativo de la inmigración en los medios de comunicación españoles. Un análisis de contenido desde la teoría del framing". Estudios sobre el Mensaje Periodístico 13: 91-110.

Izurieta, Ana. 2018. "Análisis de la Ley Orgánica de Movilidad Humana". Tesis de licenciatura, Universidad San Francisco de Quito.

Lakoff, George, y Mark Johnson. 2015. Metáforas de la vida cotidiana. Madrid: Cátedra.

Llamas, Valeria. 2016. “Seguridad humana y movilidad humana”. Revista IIDH 63: 147-85.

Massoni, Sandra. 2013a. “Comunicación estratégica: Matrices de datos en la investigación enactiva”. Revista Latinoamericana de Ciencias de la Comunicación 10 (18): 90-102.

-. 2013b. Metodologías de la comunicación estratégica: Del inventario al encuentro sociocultural. Santa Fe, AR: Homo Sapiens. 
-.2016. Avatares del comunicador complejo y fluido. Del perfil del comunicador social y otros devenires. Quito: CIESPAL.

-. 2018. "Investigación enactiva en comunicación, metodologías participativas y asuntos epistemológicos". Revista Latinoamericana de Ciencias de la Comunicación 28: 83-93.

—, Mariana Piola, y Mateo Bussi. 2017. “Un sistema de medición comunicacional más allá del control. Indicadores comunicacionales en dimensiones múltiples”. En Repensando la estrategia desde la comunicación, editado por Amaia Arribas, Raúl Herrera y Rafael Pérez, 202-29. Quito: Razón y Palabra.

Ortiz, Loretta. 2018. Derecho internacional público. Oxford, GB: Oxford University Press.

Ponte, Jorge. 2014. "La prensa como vehículo de representaciones sociales en tiempos del modernismo (1885-1910). Un ejemplo de aplicación: La ciudad de Mendoza (Argentina)”. TINKUY 21: 109-28.

Rubira, Rainer, y Belén Puebla. 2018. "Representaciones sociales y comunicación: Apuntes teóricos para un diálogo interdisciplinar inconcluso". Convergencia 76: 147-67.

Ruiz-Aranguren, María. 2018. "La inmigración en la esfera mediática”. Communication \& Society 31 (2): 87-100.

Van Dijk, Teun. 1999. “Análisis crítico del discurso”. Anthropos 186: 23-36.

-. 2006. Ideología. Barcelona: Gedisa.

Vásquez, Rodrigo, Carlos Castillo, y Lydia Lera. 2015. "Migraciones en países de América Latina.

Características de la población pediátrica”. Revista Chilena de Pediatría 86 (5): 325-30.

Verón, Eliseo. 1981. Construir el acontecimiento. Barcelona: Gedisa. 